OPEN ACCESS

Edited by:

Dong-Yuan Cao,

Xi'an Jiaotong University, China

Reviewed by:

Peijing Rong,

China Academy of Chinese Medical

Sciences, China

Chun Lin,

Fujian Medical University, China

Chao Bian,

University of Maryland, Baltimore,

United States

*Correspondence:

Yong Tang

tangyong@cdutcm.edu.cn

tThese authors have contributed equally to this work

Specialty section:

This article was submitted to

Perception Science,

a section of the journal

Frontiers in Neuroscience

Received: 18 April 2021

Accepted: 27 August 2021

Published: 28 September 2021

Citation:

Tahir AH, Li J-J and Tang Y (2021) Peripheral and Spinal Mechanisms Involved in Electro-Acupuncture

Therapy for Visceral Hypersensitivity.

Front. Neurosci. 15:696843.

doi: 10.3389/fnins.2021.696843

\section{Peripheral and Spinal Mechanisms Involved in Electro-Acupuncture Therapy for Visceral Hypersensitivity}

\author{
Adnan Hassan Tahir ${ }^{1,2+}$, Jia-Jia $\mathrm{Li}^{1,2+}$ and Yong Tang ${ }^{1,2 *}$ \\ ${ }^{1}$ School of Acupuncture and Tuina and International Collaborative Centre on Big Science Plan for Purinergic Signalling, \\ Chengdu University of Traditional Chinese Medicine, Chengdu, China, ${ }^{2}$ Acupuncture and Chronobiology Key Laboratory \\ of Sichuan Province, Chengdu, China
}

One of the important characteristic features of clinically significant gastrointestinal disorders is visceral hypersensitivity $(\mathrm{VH})$. Pain sensitization or $\mathrm{VH}$ is a big challenge for clinicians and becomes a very thorny work in clinical practices; the therapeutic efficacy for $\mathrm{VH}$ results in limited success. A popular second therapy that is being approved for the induction of analgesia and attenuates $\mathrm{VH}$ with fewer side effects includes electroacupuncture (EA). Different peripheral and spinal neurological chemicals, including neurotransmitters, neuropeptides, and cytokines, and different signaling pathways were associated with EA treatment in $\mathrm{VH}$. Despite the higher acceptance of EA, the underlying mechanism still needs to be further explored. In this paper, we review the available literature to find the peripheral and spinal mechanisms involved in $\mathrm{EA}$ to relieve $\mathrm{VH}$.

\footnotetext{
Keywords: visceral hypersensitivity, electro-acupuncture analgesia, peripheral mechanism, spinal cord, neurological chemicals
}

\section{INTRODUCTION}

The definition of visceral hypersensitivity $(\mathrm{VH})$ includes hypersensitive visceral pain perception to colorectal distension (CRD), which is an experience by humans and animals having functional gastrointestinal (GI) disorders, such as inflammatory bowel disease (IBD) and irritable bowel syndrome (IBS) (Shah et al., 2016; Botschuijver et al., 2017). The mechanism involved in the pathogenesis of VH in IBD includes the spontaneous release of inflammatory mediators predominantly under the progression of an inflammatory attack and the recurrence that in turn causes sensitization of peripheral nerves in the enteric wall (Minderhoud et al., 2004; Bielefeldt et al., 2009). One of the most influencing factors causing abdominal pain in IBS is VH that is the contributing cause of triggering pain sensation in the bowel (Barbara et al., 2011). VH is a biological marker for IBS. The occurrence of VH in such long-term intestinal disorders has been known to cause and increase mortality rate and irreparable loss of the quality of life of humans and animals, resulting in a deem loss of economy on the worldwide basis (Zielińska et al., 2019). Hence, the underlying contributing factors include specific inflammatory processes, environmental, psychological, genetic, and microbiological parameters responsible for causing VH in IBD and IBS, but the exact mechanism of the cause of $\mathrm{VH}$ in these particular disorders is still unknown.

The clinical help seeking patients having IBS or IBD hold the consistent progression of VH. The pharmacological approach for the treatment of $\mathrm{VH}$ includes analgesics (non-steroidal antiinflammatory drugs), antispasmodics, and antidepressants. Still, the use of such medications has been known to cause certain side effects that include insufficient pain relief, tolerance, and GI 
and cardiovascular toxicities (Davis, 2012). Some alternative therapies, including acupuncture, have been known to be effective treatment protocols that possess efficient therapeutic effects and minimum side effects compared with pharmacotherapy. As far as acupuncture is concerned, it can be defined as an oldest Chinese healing therapy that has been extensively used treatment protocol for humans and animals for over the last 3,000 years. Now, the modern Western medicine has also been convinced with the similarities as shown by the Chinese medicine (Bivins, 2001). In the present world, acupuncture therapy has been demonstrated to be a significantly popular and effective therapeutic for various diseases showing resistance to the conventional modes of treatment (Xie and Ortiz-Umpierre, 2006; Hutchinson et al., 2012).

Past studies have shown the relief of pain in response to acupuncture therapy as compared with the sham acupuncture or control groups. Acupuncture treatment is being associated with the change in the concentrations of $\beta$-endorphin, epinephrine, and serotonin and the levels of c-Fos, substance $\mathrm{P}$, cytokines, P2X3, acetylcholine (Ach), N-methyl-D-aspartate (NMDA) receptors, and serotonin in the gut/spinal cord (Wan et al., 2017; Lee et al., 2019; Tan et al., 2019; Zhang et al., 2020). These studies show that electro-acupuncture (EA) can be used as an effective therapy to relieve $\mathrm{VH}$. It is necessary to find more conclusive information to verify the effectiveness of EA and understand its basic mechanism. This review paper will summarize the effectiveness of EA to attenuate $\mathrm{VH}$ and try to find a new era of study in this field. Peripheral and spinal neurobiological mechanisms are involved in the attenuation of $\mathrm{VH}$ in different animal models by using acupuncture (Figure 1).

\section{ELECTRO-ACUPUNCTURE STIMULUS IN SKIN COULD REGULATE NOCICEPTIVE PROCESSING IN INTESTINES AND SPINAL CORD}

EA is a modified form of traditional manual acupuncture (MA), in which it applies electrical stimulation to the peripheral nerves through the inserted needles at the acu-points. Most researchers use EA to prevent and treat different disorders because it can be easily standardized by voltage, frequency, and wavelength, it has better effects, and its stimulation can be objectively controlled and quantified. EA with different intensities can excite different types of peripheral afferent fibers and produce different extents of analgesia. The transmission of visceral nociception could be inhibited by EA in an intensitydependent manner (Yu et al., 2019). EA analgesic effects are mediated through $\mu$-and $\delta$-opioid receptors at the lower frequency $(2 \mathrm{~Hz})$ and $\kappa$-opioid receptors at the higher frequency $(100 \mathrm{~Hz})$. At the two $\mathrm{Hz}$ frequencies, EA stimulates the release of $\beta$-endorphins, endomorphin, and encephalin. At $100 \mathrm{~Hz}$, it releases dynorphin in the central nervous system (CNS) and binds to their corresponding receptors (Han, 2003, 2004). It is generally established that EA achieves analgesic effects on visceral pain via somatovisceral interactions on convergent neurons at different levels of the CNS (Wang et al., 2014). The wide dynamic range (WDR) neurons of the spinal dorsal horn could play an important role in managing visceral nociception (Zhang et al., 2011). These neurons receive a convergence of inputs from the external environment (the skin) and the internal milieu (the viscera, muscles, etc.) (Le Bars, 2002).

\section{PERIPHERAL MECHANISM OF ELECTRO-ACUPUNCTURE FOR REDUCING VISCERAL HYPERSENSITIVITY}

The present studies have shown that one of the promising conditions of visceral GI pain is VH. The peripheral and central mechanisms are involved in the pathogenesis of $\mathrm{VH}$. The past studies have depicted that various harmful stimuli play a key role by affecting the receptors of the enteric mucosal linings resulting in the alertness of mast cells to secrete various inflammatory mediators, such as prostaglandins and bradykinin, thereby acting on the respective receptors of the sensory nerve endings (Mayer and Tillisch, 2011). Thus, nociceptive responses are transmitted to the spinal cord. The basic feature to study the neurological mechanism of EA for the relief of GI visceral pain has involved the study of neurons and the corresponding neurotransmitters and afferent fibers.

Acupuncture regulates the different peripheral neurobiological chemicals, including neurotransmitters, peptides, and cytokines, which cause to attenuate $\mathrm{VH}$. In the intrinsic nervous system, cholinergic neurons of the intestinal myenteric plexus and sub-mucosal nerve plexus released an important neurotransmitter called Ach. Ach is considered as a significant neurotransmitter for regulating GI motility (McConalogue and Furness, 1994) and a powerful mediator of intestinal function (Yoo and Mazmanian, 2017), contributing to the primary afferents of acupuncture analgesic mechanism. Acupuncture may relieve visceral pain response by reducing the Ach level, which increased during inflammatory reactions (Yang et al., 2010).

The expression of substance $\mathrm{P}$ significantly lowered after using acupuncture (Liu et al., 2010; Yang et al., 2010). Substance $\mathrm{P}$ has also been known to play a key role in the progression of acupuncture analgesia (Karatay et al., 2018). EA at acupoint ST25 and ST37 significantly decreased VH response and mast cells count, substance $\mathrm{P}$, neurokinin, certain vasoactive amines, and peptides in the colon of an IBS rat model induced with mechanical colorectal irritation (Wu et al., 2008; Xiao-Peng et al., 2009).

Another neurotransmitter serotonin [5-hydroxytryptamine (5-HT)] in the CNS and digestive tract regularizes various features of the digestive system. 5-HT and its receptors have been predominantly shown in the myenteric nerve plexus and participate in the different functions of the digestive tract (Huang et al., 2014). Previous research found an increase of 5-HT and its receptors in the intestine of patients suffering $\mathrm{VH}$ (Holtmann et al., 2016). 5-HT is involved in the regulation of 


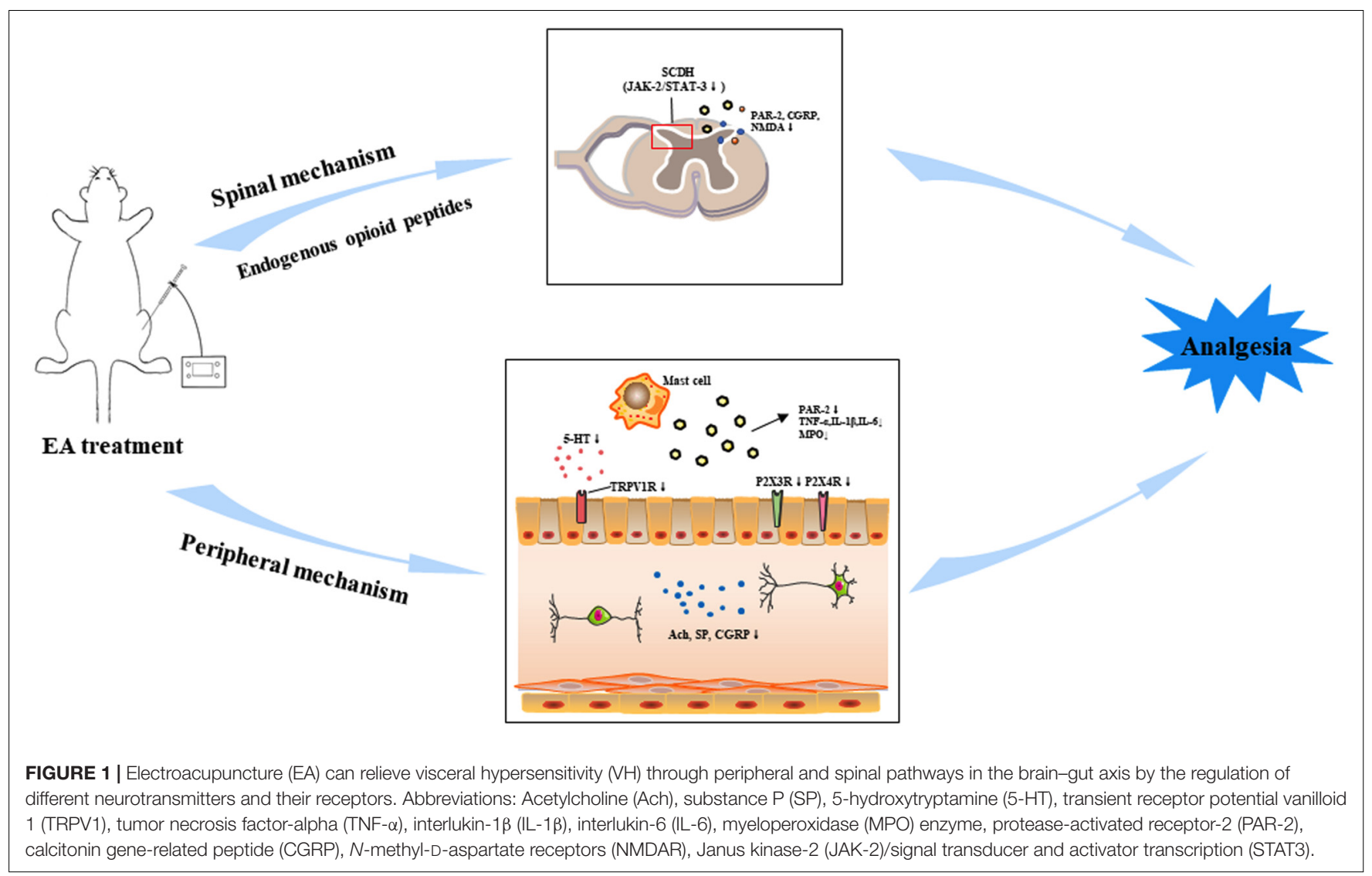

activation of the transient receptor potential vanilloid 1 (TRPV1) (Winston et al., 2007). Thus, for the effective therapeutic treatment in $\mathrm{VH}$-associated disorders, 5-HT is known to play a key role (Dunlop et al., 2005). EA stimulation decreased the colonic levels of 5-HT and 5-HT3 receptor expression in the brain-gut axis (Zhao et al., 2016). EA bilaterally at acupoint ST36 prominently lowered VH and colon 5-HT3 receptors expression in the IBS model induced by intrarectal acetic acid injection (Chu et al., 2011).

Different cytokines like tumor necrosis factor-alpha (TNF- $\alpha$ ), interlukin-1 $\beta$ (IL-1 $\beta$ ), and interlukin-6 (IL-6) and disease activity index, histopathological changes scour, and myeloperoxidase (MPO) enzyme expression increase in the inflamed intestine and have an important role in VH (Adam et al., 2013; Shah et al., 2016; Tahir et al., 2016). After acupuncture treatment in the 2,4,6trinitrobenzene sulfonic acid (TNBS)-induced colitis or ileitis model, the level of TNF- $\alpha$, IL- $1 \beta$, and IL- 6 and disease activity index, microscopic changes scour, and MPO level were decreased and caused to attenuate $\mathrm{VH}$, and weight gain was improved (Tian et al., 2003; Kim et al., 2017; Shah et al., 2020).

A previous study showed that protease-activated receptor2 (PAR-2) activation in different cells like granulocytes and certain agranulocytic white blood cells could induce the release of different inflammatory mediators, i.e., chemokines and prostaglandins, which promoted the production of neurogenic mediators, i.e., substance $P$ and calcitonin gene-related peptide (CGRP), from the enteric nerve plexus and afferent nerves to ultimately trigger the VH (Cenac et al., 2003; Suckow and Caudle, 2009). Fecal supernatant of IBS patients was injected into the colon of healthy mice that resulted in higher mucosal inflammation and increased intestinal permeability, hence contributing to VH through a PAR-2 activation mechanism (Cenac et al., 2007; Annaházi et al., 2009; Shah et al., 2020). The respective findings have shown that activated PAR-2 in the intestine enhances $\mathrm{VH}$. EA may relieve $\mathrm{VH}$ by reducing the mast cells number and the expression of PAR-2, substance P, CGRP, TRPV1, Toll-like receptor 4, and tryptase proteins in the colon tissue of IBS rats (Deng et al., 2018; Yang et al., 2019; Chen et al., 2021).

After the discovery of purinergic receptors by Burnstock, it is experimentally verified that adenosine triphosphate (ATP) is released from the epithelial cells of tubular organs or saclike organs after mechanical distension stimulation; this stimulation acts on the nerve plexus of the purinergic receptor P2X under the epithelial mucosa, thereby causing the transmission of pain signals to the pain center (Burnstock and Kennedy, 2011; Burnstock, 2014a). This assertion has been demonstrated in many GI disorders, such as IBS, IBD, and interstitial cystitis (Burnstock, 2014b). P2X receptors have been widely distributed in the body and are known to be involved in the formation, transduction, and regulation of neuropathic pain, inflammatory pain, and visceral pain or VH. Burnstock suggested that acupuncture mainly acts through purinergic signals (Burnstock, 2011). Purinergic signaling has recently been suggested to constitute the 
cellular mechanism underlying acupuncture-induced analgesia. By extending the original hypothesis on endogenous opioids being released during acupuncture analgesia, Burnstock and Nedergaard provided evidence for the involvement of purinergic receptors (P2 and $\mathrm{P} 1 / \mathrm{A} 1$ receptors) in the beneficial effects of acupuncture-induced analgesia (Tang et al., 2018). This statement provided new ideas for the study of the mechanism involving acupuncture and depicted new challenges (Huang et al., 2021).

A previous study demonstrated that EA could regulate the expression of the $\mathrm{P} 2 \mathrm{X} 3$ receptors in the peripheral and central pathways of visceral pain transmission to achieve remission of $\mathrm{VH}$ in the IBS rat model (Weng et al., 2015). EA downregulated the increased expression of $\mathrm{P} 2 \mathrm{X} 3$ receptors in the colonic myenteric plexus and colon-associated dorsal root ganglion (DRG) neurons and is involved in the attenuation of $\mathrm{VH}$ in rats with the IBS model (Zhang et al., 2020). EA can attenuate VH in the IBS rat model by decreasing the expression of $\mathrm{P} 2 \mathrm{X} 4$ receptors in the colon (Guo et al., 2013). There is further need to explore the mechanism of EA on the brain-gut neural signal transmission at different levels, including the enteric nervous system in $\mathrm{VH}$, and investigate the other P2X and P2Y receptors. Therefore, it can be said that EA can downregulate different peripheral chemicals to lower the sensitization process of the enteric nerves and have the effect to alleviate VH (Figure 1). Despite that fact, there is a vital need to investigate the new experimental designs that concern the specific agonists, antagonists, and gene knockouts to find more convincing information to understand the peripheral neurobiological mechanism of EA involved in the attenuation of $\mathrm{VH}$.

\section{SPINAL MECHANISM OF ELECTRO-ACUPUNCTURE FOR REDUCING VISCERAL HYPERSENSITIVITY}

There is a strong covenant that $\mathrm{VH}$ is associated with spinal cord sensitization, which involves different excitatory neurotransmitters (Bueno et al., 1997; Khan et al., 2006; Kapural et al., 2010; Baranidharan et al., 2014). In the spinal cord dorsal horn (SCDH), different vital afferent nerves converge from the periphery, SCDH neurons, and descending nerves from the superior center, thereby forming a neuron network having complex characteristics. The spinal cord is composed of various neurotransmitters and the corresponding receptors, certain neuromodulators, and various ion channels that are involved in the reception and transmission of nociceptive information (Luo et al., 2014; Zhang et al., 2017). Inhibition of central sensitization causes to alleviate visceral pain or VH (Sarkar et al., 2001).

Acupuncture stimulation may inhibit the neural response activated by enteric nociceptive afferents and be responsible for the induction of the attenuation of visceral pain or $\mathrm{VH}$ (Yu et al., 2014). A previous study found that neuromodulators, especially some endogenous opioid peptides (endorphin, enkephalin, and dynorphin), have significant contributions in EA analgesia (Przewłocki et al., 1986). EA can boost the release of different opioids, which act on their related receptors to exert an analgesic effect. EA can inhibit the effect of different neurotransmitters involved in pain sensitization by regulating endogenous opioids in the DRG and spinal cord, resulting in the attenuation of visceral pain response (Qi et al., 2016).

c-Fos has been exclusively considered a biological indicator to examine the neuronal activity, as the expression of c-Fos boost in the spinal cord causes the excitement of the CNS. The continuous or subsequent nociceptive stimuli increase spinal cord c-Fos expression, contributing to the generation of a pain state partly because of adaptive spinal response (Qiu et al., 2015). Therefore, c-Fos is providing the active site for evaluating the active substances or treatment in the CNS. Researchers have also established that EA reduced peripheral inflammation and c-Fos expression in the spinal cord (Xu et al., 2010; Qi and Li, 2012; Shah et al., 2020). The c-Fos expression level increased in the SCDH superficial lamina during $\mathrm{VH}$ with TNBS-induced ileitis but decreased after EA therapy (Shah et al., 2020). This phenomenon indicating that ileitis-induced $\mathrm{VH}$ activated the SCDH superficial lamina was the important site on which EA regulates its effects. This indication shows that EA inhibits the transmission of noxious stimulation at the spinal cord level, which may involve the participation of different neurobiological molecules. EA reduces the expression of c-Fos, p38, and 5-HT receptors and ultimately causes the attenuation of pain sensitization (Wu et al., 2010; Xu et al., 2010). EA can reduce the level of $\mathrm{c}$-Fos and different NMDA excitatory receptors in the spinal cord and attenuate VH (Tian et al., 2008; Wang et al., 2009).

The agonist of PAR-2 enhances the potassium chloride ion and CGRP in the sensory neuron (Steinhoff et al., 2000; Hoogerwerf et al., 2001). The role of CGRP in VH has been confirmed by using CGRP gene knockout and antagonist of CGRP receptor in rodents (Delafoy et al., 2006; Thompson et al., 2008). EA can attenuate $\mathrm{VH}$ by reducing the expression of c-Fos, PAR-2, and CGRP in the SCDH in the rats with IBD and IBS models (Sun et al., 2015; Shah et al., 2020).

The Janus kinase (JAK)/signal transducer and activator transcription (STAT) pathway activated by cytokines is the key signaling mechanism in central sensitization. JAK-2 specifically activates the STAT3 in the spinal cord and involves VH (Sriram et al., 2004; Bradesi et al., 2015). IL-6 is one of the important up-streaming pro-inflammatory cytokines of the JAK-2/STAT3 pathway. In goats with TNBS-induced ileitis, TNBS enhanced the visceral motor response (VMR) and pain behavior response to CRD and increased the expression of IL-6, JAK-2, and STAT3 in the SCDH, periaqueductal gray (PAG), and rostral ventromedial medulla (RVM). EA treatment attenuated the VMR and pain behavior response and decreased the IL-6, JAK-2, and STAT3 expression in the SCDH, PAG, and RVM (Wan et al., 2017). EA relieved the $\mathrm{VH}$ primarily by inhibiting the JAK-2/STAT3 signaling pathway in the PAG-RVM-SCDH axis (Wan et al., 2017).

It is known that for approximately 50 years, the nucleotides are known to perform a critical role in the induction of nociception and have an essential role in the mechanisms of pain (Burnstock, 2016). Extracellular ATP induces its downregulation 
signaling through activation of two major P2 purinergic receptors. $\mathrm{P} 2 \mathrm{X}$ is the ionotropic, and $\mathrm{P} 2 \mathrm{Y}$ is the metabotropic $G$ protein-coupled receptors. As we discussed previously, the purinergic receptors in the peripheral and CNS are involved in the process of visceral hypersensitization in IBS. Acupuncture may attenuate the $\mathrm{VH}$ by regulating the purinergic receptors. EA can attenuate $\mathrm{VH}$ by reducing purinergic $\mathrm{P} 2 \mathrm{X} 3$ receptor expression in the intestine and CNS by regulating brain-gut neural signal transmission (Weng et al., 2015). Still, there is a need to find a more detailed neural mechanism by which EA is involved in the attenuation of $\mathrm{VH}$ in the spinal cord under purinergic signaling molecules.

\section{CONCLUSION}

EA mechanisms involved in the attenuation of $\mathrm{VH}$ have been extensively studied. This review demonstrated the attenuation of $\mathrm{VH}$ following EA treatment. The EA treatment causes inhibition of both the sensory and respective affective components of $\mathrm{VH}$ via the routes of the peripheral and spinal cord, by the involvement of aggregation of bioactive neurobiological molecules including opioids, cytokines (TNF$\alpha$, IL-1 $\beta$, and IL-6), serotonin, substance P, NK1, PAR-2, CGRP, glutamatergic NMDA receptors, purinergic $\mathrm{P} 2 \mathrm{X} 3$ receptor, P38, JAK-2, and STAT3 signaling molecules (Figure 1). How these neurobiological molecules and structures (SCDH and visceral organs) combined work together remains unidentifiable. Purinergic signaling molecules are more recent players in the field of EA analgesia (Tang et al., 2018; He et al., 2020), but only few studies are available about the analgesia effects of EA on visceral organs. Still, there is a need to find a more detailed neural mechanism by which EA is involved in the attenuation of $\mathrm{VH}$

\section{REFERENCES}

Adam, B., Tsopelas, C., Liebregts, T., Bartholomeusz, F. D., and Holtmann, G. (2013). Host immune response determines visceral hyperalgesia in a rat model of post-inflammatory irritable bowel syndrome. J. Gastroenterol. 48, 1119-1127. doi: 10.1007/s00535-012-0729-2

Annaházi, A., Gecse, K., Dabek, M., Ait-Belgnaoui, A., Rosztóczy, A., Róka, R., et al. (2009). Fecal proteases from diarrheic-IBS and ulcerative colitis patients exert opposite effect on visceral sensitivity in mice. Pain 144, 209-217. doi: 10.1016/j.pain.2009.04.017

Baranidharan, G., Simpson, K. H., and Dhandapani, K. (2014). Spinal cord stimulation for visceral pain-a novel approach. Neuromodulation 2014, $753-$ 758. doi: 10.1111/ner.12166

Barbara, G., Cremon, C., De Giorgio, R., Dothel, G., Zecchi, L., Bellacosa, L., et al. (2011). Mechanisms underlying visceral hypersensitivity in irritable bowel syndrome. Curr. Gastroenterol. Rep. 13, 308-315. doi: 10.1007/s11894-0110195-7

Bielefeldt, K., Davis, B., and Binion, D. G. (2009). Pain and inflammatory bowel disease. Inflamm. Bowel Dis. 15, 778-788. doi: 10.1002/ibd.20848

Bivins, R. (2001). The needle and the lancet: acupuncture in Britain, 1683-2000. Acupunct. Med. 19, 2-14. doi: 10.1136/aim.19.1.2

Botschuijver, S., Roeselers, G., Levin, E., Jonkers, D. M., Welting, O., Heinsbroek, S. E. M., et al. (2017). Intestinal fungal dysbiosis is associated with visceral hypersensitivity in patients with irritable bowel syndrome and rats. Gastroenterology 153, 1026-1039. doi: 10.1053/j.gastro.2017.06.004 in the intestine and spinal cord under the purinergic signaling pathway. There is also a need to investigate the new experimental designs that concern the specific agonists, antagonists, and gene knockouts to find more convincing information to understand the peripheral and spinal neurobiological mechanisms of EA involved in the attenuation of $\mathrm{VH}$. Additionally, the intestinal microbiome is recognized to be responsible for the control of VH (Li et al., 2020; West and Neufeld, 2021). It is very necessary to punch the truth whether the intestinal microbiome or gutbrain-microbiota axis (Yaklai et al., 2021) plays a crucial role in acupuncture reducing $\mathrm{VH}$. Another recent report indicated that inhibiting the activation of astrocytes in the medial thalamus and anterior cingulate cortex would get involved in EA alleviating IBS VH. It implied that the central mechanism should not be neglected in the future research (Zhao et al., 2020).

\section{AUTHOR CONTRIBUTIONS}

AT and J-JL drafted the manuscript. All authors discussed, edited, and approved the final version for submission.

\section{FUNDING}

This work was supported by grants from the National Key R\&D Program of China (2019YFC1709101), the National Natural Science Foundation of China (81373735), the Project First-Class Disciplines Development of Chengdu University of Traditional Chinese Medicine (CZYHW1901), the MDT program of the State Administration of Traditional Chinese Medicine, and the Science and Technology Program of Sichuan Province, China (2019YFH0108).

Bradesi, S., Karagiannides, I., Bakirtzi, K., Joshi, S. M., Koukos, G., Iliopoulos, D., et al. (2015). Identification of spinal cord MicroRNA and gene signatures in a model of chronic stress-induced visceral hyperalgesia in rat. PLoS One 10:e0130938. doi: 10.1371/journal.pone.0130938

Bueno, L., Fioramonti, J., Delvaux, M., and Frexinos, J. (1997). Mediators and pharmacology of visceral sensitivity: from basic to clinical investigations. Gastroenterology 112, 1714-1743. doi: 10.1016/S0016-5085(97)70056-8

Burnstock, G. (2011). Puncturing the myth. Scientist 25, 24-25.

Burnstock, G. (2014a). Purinergic signalling in the gastrointestinal tract and related organs in health and disease. Purinergic Signal. 10, 3-50. doi: 10.1007/s11302013-9397-9

Burnstock, G. (2014b). Purinergic signalling in the urinary tract in health and disease. Purinergic Signal. 10, 103-155. doi: 10.1007/s11302-0139395-y

Burnstock, G. (2016). Purinergic mechanisms and pain. Adv. Pharmacol. 75, 91-137. doi: 10.1016/bs.apha.2015.09.001

Burnstock, G., and Kennedy, C. (2011). P2X receptors in health and disease. Adv. Pharmacol. 61, 333-372. doi: 10.1016/B978-0-12-385526-8.00011-4

Cenac, N., Andrews, C. N., Holzhausen, M., Chapman, K., Cottrell, G., AndradeGordon, P., et al. (2007). Role for protease activity in visceral pain in irritable bowel syndrome. J. Clin. Invest. 117, 636-647. doi: 10.1172/JCI29255

Cenac, N., Garcia-Villar, R., Ferrier, L., Larauche, M., Vergnolle, N., Bunnett, N. W., et al. (2003). Proteinase-Activated receptor-2-induced colonic inflammation in mice: possible involvement of afferent neurons, nitric oxide, and paracellular permeability. J. Immunol. 170, 4296-4300. doi: 10.4049/jimmunol.170.8.4296 
Chen, Y., Cheng, J., Zhang, Y., Chen, J. D. Z., and Seralu, F. M. (2021). Electroacupuncture at ST36 relieves visceral hypersensitivity via the NGF/TrkA/TRPV1 peripheral afferent pathway in a rodent model of post-inflammation rectal hypersensitivity. J. Inflamm. Res. 14, 325-339.

Chu, D., Cheng, P., Xiong, H., Zhang, J., Liu, S., and Hou, X. (2011). Electroacupuncture at ST-36 relieves visceral hypersensitivity and decreases 5HT3 receptor level in the colon in chronic visceral hypersensitivity rats. Int. J. Colorectal Dis. 26, 569-574. doi: 10.1007/s00384-010-1087-2

Davis, M. P. (2012). Drug management of visceral pain: concepts from basic research. Pain Res. Treat. 2012, 265605. doi: 10.1155/2012/265605

Delafoy, L., Gelot, A., Ardid, D., Eschalier, A., Bertrand, C., Doherty, A. M., et al. (2006). Interactive involvement of brain derived neurotrophic factor, nerve growth factor, and calcitonin gene related peptide in colonic hypersensitivity in the rat. Gut 55, 940-945. doi: 10.1136/gut.2005.064063

Deng, D. X., Tan, J., Zhang, H., Huang, G. L., Li, S., Guo, K. K., et al. (2018). Electroacupuncture relieves visceral hypersensitivity by down-regulating mast cell number, PAR-2/TRPV 1 signaling, etc. in colonic tissue of rats with irritable bowel syndrome. Acupunct. Res. 43, 485-491.

Dunlop, S. P., Coleman, N. S., Blackshaw, E., Perkins, A. C., Singh, G., Marsden, C. A., et al. (2005). Abnormalities of 5-hydroxytryptamine metabolism in irritable bowel syndrome. Clin. Gastroenterol. Hepatol. 3, 349-357. doi: 10.1016/ S1542-3565(04)00726-8

Guo, X. X., Chen, J. F., Lu, Y., Wu, L. Y., Weng, Z. J., Yang, L., et al. (2013). Electroacupuncture at $\mathrm{He}-\mathrm{Mu}$ points reduces $\mathrm{P} 2 \mathrm{X} 4$ receptor expression in visceral hypersensitivity. Neural Regen. Res. 8, 2069-2077. doi: 10.3969/j.issn. 1673-5374.2013.22.006

Han, J. S. (2003). Acupuncture: neuropeptide release produced by electrical stimulation of different frequencies. Trends Neurosci. 26, 17-22. doi: 10.1016/ S0166-2236(02)00006- 1

Han, J.-S. (2004). Acupuncture and endorphins*1. Neurosci. Lett. 361, 258-261. doi: 10.1016/j.neulet.2003.12.019

He, J. R., Yu, S. G., Tang, Y., and Illes, P. (2020). Purinergic signaling as a basis of acupuncture-induced analgesia. Purinergic Signal. 16, 297-304. doi: 10.1007/ s11302-020-09708-z

Holtmann, G. J., Ford, A. C., and Talley, N. J. (2016). Pathophysiology of irritable bowel syndrome. Lancet Gastroenterol. Hepatol. 1, 133-146. doi: 10.1016/ S2468-1253(16)30023-1

Hoogerwerf, W. A., Zou, L., Shenoy, M., Sun, D., Micci, M. A., Lee-Hellmich, H., et al. (2001). The proteinase-activated receptor 2 is involved in nociception. J. Neurosci. 21, 9036-9042. doi: 10.1523/jneurosci.21-22-09036.2001

Huang, R., Zhao, J., Wu, L., Dou, C., Liu, H., Weng, Z., et al. (2014). Mechanisms underlying the analgesic effect of moxibustion on visceral pain in irritable bowel syndrome: a review. Evid. Based Complement. Altern. Med. 2014:895914. doi: 10.1155/2014/895914

Huang, Z., Xie, N., Illes, P., Virgilio, F. D., Ulrich, H., Semyanov, A., et al. (2021). From purines to purinergic signalling: molecular functions and human diseases. Signal Transduct. Target. Ther. 6:162.

Hutchinson, A. J. P., Ball, S., Andrews, J. C. H., and Jones, G. G. (2012). The effectiveness of acupuncture in treating chronic non-specific low back pain: a systematic review of the literature. J. Orthop. Surg. Res. 7, 36-43. doi: 10.1186/ 1749-799X-7-36

Kapural, L., Nagem, H., Tlucek, H., and Sessler, D. I. (2010). Spinal cord stimulation for chronic visceral abdominal pain. Pain Med. 11, 347-355. doi: 10.1111/j. 1526-4637.2009.00785.x

Karatay, S., Okur, S. C., Uzkeser, H., Yildirim, K., and Akcay, F. (2018). Effects of acupuncture treatment on fibromyalgia symptoms, serotonin, and substance P levels: a randomized sham and placebo-controlled clinical trial. Pain Med. (United States) 19, 615-628. doi: 10.1093/pm/pnx263

Khan, Y. N., Raza, S. S., and Khan, E. A. (2006). Spinal cord stimulation in visceral pathologies. Pain Med. 7, S121-S125. doi: 10.1111/j.1526-4637.2006.00127.x

Kim, D. H., Ryu, Y., Hahm, D. H., Sohn, B. Y., Shim, I., Kwon, O. S., et al. (2017). Acupuncture points can be identified as cutaneous neurogenic inflammatory spots. Sci. Rep. 7:15214. doi: 10.1038/s41598-017-14359-z

Le Bars, D. (2002). The whole body receptive field of dorsal horn multireceptive neurones. Brain Res. Rev. 40, 29-44.

Lee, I. S., Cheon, S., and Park, J. Y. (2019). Central and peripheral mechanism of acupuncture analgesia on visceral pain: a systematic review. Evid. Based Complement. Altern. Med. 2019:1304152. doi: 10.1155/2019/1304152
Li, Y. J., Li, J., and Dai, C. (2020). The role of intestinal microbiota and mast cell in a rat model of visceral hypersensitivity. J. Neurogastroenterol. Motil. 26, 529-538.

Liu, J. H., Fu, W. B., Xu, Z. H., Liao, Y., and Li, X. R. (2010). [Effect of transection or pretreatment of the infraorbital nerve with snake venom on the analgesia induced by electroacupuncture of "Sibai" (ST 2) acupoint in visceral pain rats]. Zhen Ci Yan Jiu 35, 281-286.

Luo, X. Q., Cai, Q. Y., Chen, Y., Guo, L. X., Chen, A. Q., Wu, Z. Q., et al. (2014). Tyrosine phosphorylation of the NR2B subunit of the NMDA receptor in the spinal cord contributes to chronic visceral pain in rats. Brain Res. 1542, 167-175. doi: 10.1016/j.brainres.2013.10.008

Mayer, E. A., and Tillisch, K. (2011). The brain-gut Axis in abdominal pain syndromes. Annu. Rev. Med. 62, 381-396. doi: 10.1146/annurev-med-012309103958

McConalogue, K., and Furness, J. B. (1994). 3 Gastrointestinal neurotransmitters. Baillieres Clin. Endocrinol. Metab. 8, 51-76. doi: 10.1016/S0950-351X(05) 80226-5

Minderhoud, I. M., Oldenburg, B., Wismeijer, J. A., Van Berge Henegouwen, G. P., and Smout, A. J. P. M. (2004). IBS-like symptoms in patients with inflammatory bowel disease in remission; relationships with quality of life and coping behavior. Dig. Dis. Sci. 49, 469-474. doi: 10.1023/B:DDAS.0000020506. 84248.f9

Przewłocki, R., Lasoń, W., Silberring, J., Herz, A., and Przewłocka, B. (1986). Release of opioid peptides from the spinal cord of rats subjected to chronic pain. NIDA Res. Monogr. 75, 422-425.

Qi, D. B., and Li, W. M. (2012). Effects of electroacupuncture on expression of c-fos protein in the spinal dorsal horn of rats with chronic visceral hyperalgesia. J. Chin. Integr. Med. 10, 1490-1496. doi: 10.3736/jcim20121224

Qi, D., Wu, S., Zhang, Y., and Li, W. (2016). Electroacupuncture analgesia with different frequencies is mediated via different opioid pathways in acute visceral hyperalgesia rats. Life Sci. 160, 64-71. doi: 10.1016/j.lfs.2016.06.025

Qiu, Z., Ding, Y., Cui, L., Hu, M., and Ding, M. (2015). The expression patterns of c-Fos and c-Jun induced by different frequencies of electroacupuncture in the brain. Evid. Based Complement. Altern. Med. 2015:343682.

Sarkar, S., Hobson, A. R., Furlong, P. L., Woolf, C. J., Thompson, D. G., and Aziz, Q. (2001). Central neural mechanisms mediating human visceral hypersensitivity. Am. J. Physiol. Gastrointest. Liver Physiol. 281, G1196-G1202.

Shah, M. K., Ding, Y., Wan, J., Janyaro, H., Tahir, A. H., Vodyanoy, V., et al. (2020). Electroacupuncture intervention of visceral hypersensitivity is involved in PAR-2-activation and CGRP-release in the spinal cord. Sci. Rep. 10:11188. doi: 10.1038/s41598-020-67702-2

Shah, M. K., Wan, J., Janyaro, H., Tahir, A. H., Cui, L., and Ding, M. (2016). Visceral hypersensitivity is provoked by 2,4,6-trinitrobenzene sulfonic acid-induced ileitis in rats. Front. Pharmacol. 7:214. doi: 10.3389/fphar.2016.00214

Sriram, K., Benkovic, S. A., Hebert, M. A., Miller, D. B., and O’Callaghan, J. P. (2004). Induction of gp130-related cytokines and activation of JAK2/STAT3 pathway in astrocytes precedes up-regulation of glial fibrillary acidic protein in the 1-methyl-4-phenyl-1,2,3,6-tetrahydropyridine model of neurodegeneration: key signaling pathway for ast. J. Biol. Chem. 279, 1993619947. doi: 10.1074/jbc.M309304200

Steinhoff, M., Vergnolle, N., Young, S. H., Tognetto, M., Amadesi, S., Ennes, H. S., et al. (2000). Agonists of proteinase-activated receptor 2 induce inflammation by a neurogenic mechanism. Nat. Med. 6, 151-158. doi: 10.1038/7 2247

Suckow, S. K., and Caudle, R. M. (2009). NMDA receptor subunit expression and PAR2 receptor activation in colospinal afferent neurons (CANs) during inflammation induced visceral hypersensitivity. Mol. Pain 5:54. doi: 10.1186/ 1744-8069-5-54

Sun, J., Wu, X., Meng, Y., Cheng, J., Ning, H., Peng, Y., et al. (2015). Electroacupuncture decreases 5-HT, CGRP and increases NPY in the brain-gut axis in two rat models of Diarrhea-predominant irritable bowel syndrome(D-IBS). BMC Complement. Altern. Med. 15:340. doi: 10.1186/s12906-015-0863-5

Tahir, A. H., Wan, J., Shah, M. K., Janyaro, H., Li, X. J., and Ding, M. X. (2016). A novel model for studying ileitis-induced visceral hypersensitivity in goats. Acta Vet. Scand. 58:72. doi: 10.1186/s13028-016-0253-0

Tan, L. H., Li, K. G., Wu, Y. Y., Guo, M. W., Lan, Y., Wang, S., et al. (2019). Effect of electroacupuncture at different acupoints on the expression of NMDA receptors in ACC and colon in IBS rats. Evid. Based Complement. Altern. Med. 2019:4213928. doi: 10.1155/2019/4213928 
Tang, Y., Yin, H. Y., Liu, J., Rubini, P., and Illes, P. (2018). P2X receptors and acupuncture analgesia. Brain Res. Bull. 151, 144-152. doi: 10.1016/j. brainresbull.2018.10.015

Thompson, B. J., Washington, M. K., Kurre, U., Singh, M., Rula, E. Y., and Emeson, R. B. (2008). Protective roles of $\alpha$-calcitonin and $\beta$-calcitonin gene-related peptide in spontaneous and experimentally induced colitis. Dig. Dis. Sci. 53, 229-241. doi: 10.1007/s10620-007-9848-7

Tian, L., Huang, Y. X., Tian, M., Gao, W., and Chang, Q. (2003). Downregulation of electroacupuncture at ST36 on TNF- $\alpha$ in rats with ulcerative colitis. World J. Gastroenterol. 9, 1028-1033. doi: 10.3748/wjg.v9.i5.1028

Tian, S. L., Wang, X. Y., and Ding, G. H. (2008). Repeated electro-acupuncture attenuates chronic visceral hypersensitivity and spinal cord NMDA receptor phosphorylation in a rat irritable bowel syndrome model. Life Sci. 83, 356-363. doi: 10.1016/j.lfs.2008.06.027

Wan, J., Ding, Y., Tahir, A. H., Shah, M. K., Janyaro, H., Li, X., et al. (2017). Electroacupuncture attenuates visceral hypersensitivity by inhibiting JAK2/STAT3 signaling pathway in the descending pain modulation system. Front. Neurosci. 11:644. doi: 10.3389/fnins.2017.00644

Wang, X., Liu, H., Ding, G., Chen, Y., Wu, H., Li, N., et al. (2009). Effects of electroacupuncture on c-Fos expression in the spinal cord and brain of rats with chronic visceral hypersensitivity. Neural Regen. Res. 4, 339-343. doi: 10.3969/j. issn.1673-5374.2009.05.003

Wang, Y., Wang, W., Li, D., Li, J., Dai, J., Liu, Y., et al. (2014). The beneficial effect of electro-acupuncture given at PC6 (Neiguan-point) by the increase in cardiac transient outward $\mathrm{K}+$ current channel which depends on the gene and protein expressions in artificially induced myocardial ischemia rats. Acupunct. Electrother. Res. 39, 259-273.

Weng, Z. J., Wu, L. Y., Zhou, C. L., Dou, C. Z., Shi, Y., Liu, H. R., et al. (2015). Effect of electroacupuncture on $\mathrm{P} 2 \mathrm{X} 3$ receptor regulation in the peripheral and central nervous systems of rats with visceral pain caused by irritable bowel syndrome. Purinergic Signal. 11, 321-329. doi: 10.1007/s11302-015-9447-6

West, C., and Neufeld, K. M. (2021). Animal models of visceral pain and the role of the microbiome. Neurobiol. Pain 10:100064.

Winston, J., Shenoy, M., Medley, D., Naniwadekar, A., and Pasricha, P. J. (2007). The vanilloid receptor initiates and maintains colonic hypersensitivity induced by neonatal colon irritation in rats. Gastroenterology. 132, 615-627. doi: 10 . 1053/j.gastro.2006.11.014

Wu, H. G., Jiang, B., Zhou, E. H., Shi, Z., Shi, D. R., Cui, Y. H., et al. (2008). Regulatory mechanism of electroacupuncture in irritable bowel syndrome: preventing MC activation and decreasing SP VIP secretion. Dig. Dis. Sci. 53, 1644-1651. doi: 10.1007/s10620-007-0062-4

Wu, J. C., Ziea, E. T., Lao, L., Lam, E. F., Chan, C. S., Liang, A. Y., et al. (2010). Effect of Electroacupuncture on visceral hyperalgesia, serotonin and fos expression in an animal model of irritable bowel syndrome. J. Neurogastroenterol. Motil. 16, 306-314. doi: 10.5056/jnm.2010.16.3.306

Xiao-Peng, M., Tan, L. Y., Yang, Y., Wu, H. G., Jiang, B., Liu, H. R., et al. (2009). Effect of electro-acupuncture on substance P, its receptor and corticotropin-releasing hormone in rats with irritable bowel syndrome. World J. Gastroenterol. 15, 5211-5217. doi: 10.3748/wjg.15.5211

Xie, H., and Ortiz-Umpierre, C. (2006). What acupuncture can and cannot treat. J. Am. Anim. Hosp. Assoc. 42, 244-248. doi: 10.5326/0420244

Xu, K. D., Liang, T., Wang, K., and Tian, D. A. (2010). Effect of preelectroacupuncture on p38 and c-Fos expression in the spinal dorsal horn of rats suffering from visceral pain. Chin. Med. J. (Engl.) 123, 1176-1181. doi: 10.3760/cma.j.issn.0366-6999.2010.09.014

Yaklai, K., Pattanakuhar, S., Chattipakorn, N., and Chattipakorn, S. C. (2021). The role of acupuncture on the gut-brain-microbiota Axis in irritable bowel syndrome. Am. J. Chin. Med. 49, 285-314.
Yang, H. Y., Guo, T. T., Ma, Y. N., Liu, T. Y., and Gao, M. (2010). [Effects of $650 \mathrm{~nm}$ laser and moxibustion pretreatment on enteric nervous system and medullary visceral zone in rats with visceral traction pain]. Zhongguo Zhen Jiu 30, 745-751.

Yang, J., Shang, B., Shi, H., Zhu, S., Lu, G., and Dai, F. (2019). The role of tolllike receptor 4 and mast cell in the ameliorating effect of electroacupuncture on visceral hypersensitivity in rats. Neurogastroenterol. Motil. 31:e13583.

Yoo, B. B., and Mazmanian, S. K. (2017). The enteric network: interactions between the immune and nervous systems of the gut. Immunity. 46, 910-926. doi: 10.1016/j.immuni.2017.05.011

Yu, L. L., Li, L., Rong, P. J., Zhu, B., Qin, Q. G., Ben, H., et al. (2014). Changes in responses of neurons in spinal and medullary subnucleus reticularis dorsalis to acupoint stimulation in rats with visceral hyperalgesia. Evid. Based Complement. Altern. Med. 2014:768634. doi: 10.1155/2014/768634

Yu, L., Wang, W., Li, L., Qin, Q., Yu, Y., Liu, K., et al. (2019). Inhibition of electroacupuncture on nociceptive responses of dorsal horn neurons evoked by noxious colorectal distention in an intensity-dependent manner. J. Pain Res. 12, 231-242. doi: 10.2147/JPR.S182876

Zhang, F., Ma, Z., Weng, Z., Zhao, M., Zheng, H., Wu, L., et al. (2020). P2X3Receptor in primary afferent neurons mediates the relief of visceral hypersensitivity by electroacupuncture in an irritable bowel syndrome rat model. Gastroenterol. Res. Pract. 2020, 8186106. doi: 10.1155/2020/8186106

Zhang, F., Wu, L., Zhao, J., Lv, T., Hu, Z., Weng, Z., et al. (2017). Neurobiological mechanism of acupuncture for relieving visceral pain of gastrointestinal origin. Gastroenterol. Res. Pract. 2017:5687496. doi: 10.1155/2017/5687496

Zhang, Y., Gong, K., Zhou, W., Shao, G., Li, S., Lin, Q., et al. (2011). Involvement of subtypes $\gamma$ and $\varepsilon$ of protein kinase $\mathrm{C}$ in colon pain induced by formalin injection. Neurosignals 19, 142-150.

Zhao, J. M., Chen, L., Zhou, C. L., Shi, Y., Li, Y. W., Shang, H. X., et al. (2016). Comparison of electroacupuncture and moxibustion for relieving visceral hypersensitivity in rats with constipation-predominant irritable bowel syndrome. Evid. Based Complement. Altern. Med. 2016:9410505. doi: 10.1155/ 2016/9410505

Zhao, M., Wang, Z., Weng, Z., Zhang, F., Li, G., Ma, Z., et al. (2020). Electroacupuncture improves IBS visceral hypersensitivity by inhibiting the activation of astrocytes in the medial thalamus and anterior cingulate cortex. Evid. Based Complement. Alternat. Med. 2020:2562979.

Zielińska, A., Sałaga, M., Włodarczyk, M., and Fichna, J. (2019). Focus on current and future management possibilities in inflammatory bowel disease-related chronic pain. Int. J. Colorectal Dis. 34, 217-227. doi: 10.1007/s00384-0183218-0

Conflict of Interest: The authors declare that the research was conducted in the absence of any commercial or financial relationships that could be construed as a potential conflict of interest.

Publisher's Note: All claims expressed in this article are solely those of the authors and do not necessarily represent those of their affiliated organizations, or those of the publisher, the editors and the reviewers. Any product that may be evaluated in this article, or claim that may be made by its manufacturer, is not guaranteed or endorsed by the publisher.

Copyright (c) 2021 Tahir, Li and Tang. This is an open-access article distributed under the terms of the Creative Commons Attribution License (CC BY). The use, distribution or reproduction in other forums is permitted, provided the original author(s) and the copyright owner(s) are credited and that the original publication in this journal is cited, in accordance with accepted academic practice. No use, distribution or reproduction is permitted which does not comply with these terms. 